\title{
Werner Ruf
}

\section{Die militärpolitische Emanzipation Deutschlands - Afrika als Exerzierplatz?}

Vorweg zur Begrifflichkeit. Der Begriff Emanzipation wurde bewusst gewählt: Er beschreibt einen Prozess. Jahrzehnte schien es undenkbar, dass deutsche Truppen wieder auf Kriegsschauplätzen auftauchen könnten. Heute sind sie wieder da, jedoch nicht im Alleingang und unter nationalem Kommando, sondern im Bündnis. Der Wandel der Rolle Deutschlands - von einem wegen des preußisch-deutschen Militarismus und zweier fürchterlicher vom Zaune gebrochener Kriege im internationalen System geächteten Paria zu einem akzeptierten, ja umworbenen Partner - ist hier kurz nachzuzeichnen. Es scheint, dass Deutschland diese neue Rolle nutzt, um im Konzert der Mächte seine eigenen Rohstoffinteressen auch militärisch abzusichern. Nicht zufällig konzentrieren sich deutsche Militäreinsätze zunehmend auf Afrika.

\section{Deutsche Außen- und Sicherheitspolitik und die Bündnisse}

Die 1949 gegründete BRD war nur bedingt souverän, sie hatte nicht einmal einen Außenminister - geschweige denn Militär. Das Misstrauen der Alliierten, vor allem der europäischen, saß tief: 1948, als die Neugründung eines (west-) deutschen Staates sich auf der internationalen Tagesordnung abzuzeichnen begann, schlossen Frankreich, Großbritannien und die Benelux-Staaten den Brüsseler Pakt, der als Verteidigungsbündnis gegen ein Wiederaufleben des deutschen Militarismus gedacht war und eine zwingende militärische Beistandspflicht enthielt - ganz im Gegensatz zu den viel weicheren Formulierungen im Artikel 5 des späteren NATO-Vertrags.

Noch der zwischen Frankreich und der jungen Bundesrepublik im Mai 1952 unterzeichnete EVG-Vertrag, der eine Wiederbewaffnung Deutschlands zur Folge gehabt hätte, scheiterte schließlich im August 1954 im französischen Parlament am Widerstand von Kommunisten und Gaullisten. Paradoxerweise eröffnete dieses Scheitern den Weg zur NATO, der die BRD 1955 beitrat.

Damit verlor der Brüsseler Pakt seinen Sinn, denn seine Mitglieder wie auch die BRD gehörten nun demselben Militärbündnis an. Der Brüsseler Pakt wurde umgewandelt in die Westeuropäische Union (WEU), der auch die BRD beitrat. Ihre Hauptaufgabe war hinfort die Überwachung der Deutschland und Italien noch auferlegten Rüstungskontrollen. Doch auch die NATO selbst 
sollte eine Kontrollfunktion über das noch immer im Geruch des aggressiven Militarismus stehende Deutschland haben: Im Gegensatz zu allen anderen Mitgliedstaaten, die Truppenteile unter nationalem Kommando und außerhalb der NATO unterhalten, wurde die gesamte Bundeswehr der NATO unterstellt. Der erste Generalsekretär der Organisation, der Brite Lord Ismay, brachte dies auf die griffige Formel, das Ziel der NATO sei „to keep the Russians out, the Americans in and the Germans down" (Schmähling 1990, 15).

Ohne hier die vielen wichtigen teils großen (z.B. Deutschlandvertrag von 1955), teils kleinen Schritte des auch ökonomischen Aufstiegs der BRD benennen zu können, ${ }^{1}$ muss eine entscheidende Etappe benannt werden - gerade weil sie in der offiziellen Erinnerungskultur der vereinten Deutschlands keine Rolle zu spielen scheint: Gemeint ist der Zwei plus Vier-Vertrag vom 12. September 1990, der den Beitritt der DDR zur BRD regelt und der die Bestimmungen des Potsdamer Abkommens von 1945 und die Verantwortung der vier Siegermächte „für Deutschland als Ganzes“ beendet. Auch dieser Vertrag ist von dem noch immer nicht erloschenen Misstrauen gegenüber dem deutschen Militarismus und seiner expansionistischen Politik geprägt: Endgültig werden (Artikel 1, Ziffer 1) die deutschen Grenzen festgeschrieben, wie sie aus dem Zweiten Weltkrieg resultieren, ausdrücklich wird die deutsch-polnische Grenze nochmals als unveränderlich bezeichnet (Ziffer 2) und unter Zustimmung beider deutscher Staaten festgelegt: Das „vereinte Deutschland hat keinerlei Gebietsansprüche gegen andere Staaten und wird solche auch in Zukunft nicht erheben“ (Ziffer 3). Und weiter (Ziffer 4): „Die Regierungen der Bundesrepublik Deutschland und der Deutschen Demokratischen Republik werden sicherstellen, dass die Verfassung des vereinten Deutschland keinerlei Bestimmungen enthalten wird, die mit diesen Prinzipien unvereinbar sind.“ Der folgende Artikel 2 ergänzt und paraphrasiert Artikel 26 des GG:

„Die Regierungen der Bundesrepublik Deutschland und der Deutschen Demokratischen Republik bekräftigen ihre Erklärungen, dass von deutschem Boden nur Frieden ausgehen wird. Nach der Verfassung des vereinten Deutschland sind Handlungen, die geeignet sind und in der Absicht vorgenommen werden, das friedliche Zusammenleben der Völker zu stören, insbesondere die Führung eines Angriffskrieges vorzubereiten, verfassungswidrig und strafbar. Die Regierungen der Bundesrepublik Deutschland und der Deutschen Demokratischen Republik erklären, dass das vereinte Deutschland keine seiner Waffen jemals einsetzen wird, es sei denn in Übereinstimmung mit seiner Verfassung und der Charta der Vereinten Nationen."

Darüber hinaus (Artikel 3) verzichtet das zu vereinigende Deutschland „auf Herstellung und Besitz von und auf Verfügungsgewalt über atomare, biologische und chemische Waffen." Eine Friedenspflicht Deutschlands scheint damit für alle kommenden Zeiten festgeschrieben. Die nun zu erörternde Frage ist, inwieweit die Bündnisse - einst zur Einhegung zumindest des westdeutschen Staates gedacht - nunmehr genutzt werden, um durch Handeln im Bündnis

1 Aus verfassungsrechtlicher Sicht hat dies Martin Kutscha (2010) nachgezeichnet. 
die verloren geglaubte und völkerrechtlich streng eingeschränkte nationale Souveränität wieder zu gewinnen.

Ein Meilenstein auf dem Weg zur Rückgewinnung militärischer Handlungsmöglichkeiten der BRD war der gut ein Jahr später geschlossene Vertrag von Maastricht (Dezember 1991). Dort beschlossen die Staaten der EU in Art. J eine Gemeinsame Außen- und Sicherheitspolitik (GASP). Militärischer Arm der GASP sollte die WEU sein, die so zu neuem Leben erweckt wurde. ${ }^{2}$ Ihre Aufgaben wurden im Juni 1992 auf dem Petersberg bei Bonn definiert („Petersberg-Aufgaben"):

- humanitäre Aufgaben,

- Rettungseinsätze,

- friedenserhaltende Aufgaben sowie

- Kampfeinsätze bei der Krisenbewältigung einschließlich friedensschaffender Maßnahmen.

Durch die Aufstellung und Zuweisung von multinationalen Verbänden (EU battle groups) sollen diese Aufgaben erfüllt werden. Auf dem EU-Gipfel in St. Malo (1998) begann die Integration der WEU in die EU, die nun auch eine Europäische Verteidigungs- und Sicherheitspolitik (ESVP) formulierte. Im Vertrag von Nizza (Dezember 2000) wurde die WEU von der EU übernommen, im Lissabon-Vertrag (Dezember 2009) wurden dann die letzten Funktionen der WEU auf die EU übertragen. Die EU verfügt damit über eine eigene Streitmacht zur Umsetzung der ESVP. Schon im Dezember 2003 hatte die EU eine eigene Sicherheitsstrategie (ESS) beschlossen (EU 2003). Sie trägt den bewegenden Titel „Ein sicheres Europa in einer besseren Welt“.

Wie jedes militärische Dokument beginnt auch die ESS mit einer Lage-Analyse:

„Seit 1990 sind fast vier Millionen Menschen - zu 90 \% Zivilisten - in Kriegen ums Leben gekommen. Weltweit haben über 18 Millionen Menschen wegen eines Konflikts ihr Heim verlassen. In weiten Teilen der dritten Welt rufen Armut und Krankheiten unsägliches Leid wie auch dringende Sicherheitsprobleme hervor. Fast drei Milliarden Menschen und damit die Hälfte der Weltbevölkerung müssen mit weniger als zwei Euro pro Tag auskommen. Jedes Jahr sterben 45 Millionen Menschen an Hunger und Unterernährung. ... Die Armut im südlich der Sahara gelegenen Teil Afrikas ist heute größer als vor zehn Jahren." (EU 2003, 2)

Die daraus gezogenen strategischen Schlussfolgerungen muten verblüffend an:

„Im Zeitalter der Globalisierung können ferne Bedrohungen ebenso ein Grund zur Besorgnis sein wie näher gelegene. ... Die erste Verteidigungslinie wird oftmals im Ausland liegen." (ESS 2003: 7)

Die Folgen des durch den global durchgesetzten Neoliberalismus verursachten sozialen Elends, der „kannibalischen Ordnung“, wie Jean Ziegler (2005) die neue Weltordnung treffend nennt, und die aus Hunger, Elend und Hass resul-

2 Art. J.4, Ziff. 2: „Die Union ersucht die Westeuropäische Union (WEU), die integraler Bestandteil der Entwicklung der Europäischen Union ist, die Entscheidungen und Aktionen der Union, die verteidigungspolitische Bezüge haben, auszuarbeiten und durchzuführen. Der Rat trifft im Einvernehmen mit den Organen der WEU die erforderlichen praktischen Regelungen.“ 
tierenden Konflikte werden auf eindimensional verkürztes militärisches Denken reduziert, zu latenten Bedrohungen stilisiert, die dann auch nur noch militärisch „bearbeitet“ werden können: Die Anwendung von Gewalt wird zwar als ultima ratio, als letztes Mittel der Konfliktlösung bezeichnet, durch die Militarisierung jeder Art von Konflikt wird aber die Anwendung militärischer Gewalt de facto zum ersten Mittel der Politik.

Betont wird allerdings in der ESS, „der grundlegende Rahmen der internationalen Beziehungen ist die Charta der Vereinten Nationen“. Jedoch: Diese diplomatische Formulierung verschleiert mehr als sie zu sagen vorgibt: Es dürfte kein Zufall sein, dass hier nicht - wie noch explizit im Zwei plus Vier-Vertrag eine Formulierung gewählt wird wie ,in Übereinstimmung mit der Charta der Vereinten Nationen“. Denn die explizit erhobene Forderung nach „präventivem Engagement" stellt eine eklatante Verletzung des Art. 2, Ziffer 4 (Gewaltverbot) der Charta der VN dar.

Dem Völkerrecht widersprechen auch die einschlägigen Regelungen zur Gemeinsamen Außen- und Sicherheitspolitik (GASP) im Lissabon-Vertrag, der am 1. Januar 2009 in Kraft trat. So bestimmt Artikel 42:

(1) „Die Gemeinsame Sicherheits- und Verteidigungspolitik ist integraler Bestandteil der Gemeinsamen Außen- und Sicherheitspolitik. Sie sichert der Union eine auf zivile und militärische Mittel gestützte Operationsfähigkeit. Auf diese kann die Union bei Missionen außerhalb der Union zur Friedenssicherung, Konfliktverhütung und Stärkung der internationalen Sicherheit in Übereinstimmung mit den Grundsätzen der Charta der Vereinten Nationen zurückgreifen."

(3) „Die Mitgliedstaaten stellen der Union für die Umsetzung der Gemeinsamen Sicherheits- und Verteidigungspolitik zivile und militärische Fähigkeiten ... zur Verfügung“. (EU 2009: 51f)

Genau wie in der ESS werden hier Kampfeinsätze außerhalb der Union (also nicht zur territorialen Verteidigung sondern zur „Friedenssicherung“!) nur in „Übereinstimmung mit den Grundsätzen“, nicht aber „,in Übereinstimmung mit der Charta“ der VN vorgesehen. Die Interpretation dieser „Grundsätze“ liegt dann bei der EU. Einsätze können vom Rat auch an „Koalitionen der Willigen" übertragen werden. Diese müssen dann nur vorher einen entsprechenden Ratsbeschluss erreichen. Im Verteidigungsfalle (Ziffer 7 des Art. 42) enthält der Vertrag einen Beistandsmechanismus, der viel umfassender ist als Art. 5 des NATO-Vertrags': „Im Falle eines bewaffneten Angriffs auf das Hoheitsgebiet eines Mitgliedstaats schulden die anderen Mitgliedstaaten ihm alle in ihrer Macht stehende Hilfe und Unterstützung ...". Dieser inzwischen unwahrscheinliche Fall entstammt noch den Regelungen jenes Brüsseler Vertrages von 1948. Worum es wirklich geht, sagt Artikel 43, der nochmals die oben

3 Art. 5 des NATO-Vertrages besagt, „dass im Falle eines Angriffs jede (Partei des Vertrages) Beistand leistet, indem jede... die Maßnahmen, einschließlich der Anwendung von Waffengewalt, trifft, die sie für erforderlich erachtet, um die Sicherheit des nordatlantischen Gebiets wieder herzustellen.“ Der Zyniker Lord Ismay kommentierte dies mit den Worten: „Im Notfall genügt eine Postkarte.“ 
erwähnten Petersberg-Aufgaben rekapituliert und in weitgehendem Einklang mit der ESS festlegt:

„Die in Artikel 42 Absatz 1 vorgesehenen Missionen ... umfassen ... Aufgaben der Konfliktverhütung und der Erhaltung des Friedens sowie Kampfeinsätze im Rahmen der Krisenbewältigung einschließlich Frieden schaffender Maßnahmen und Operationen zur Stabilisierung der Lage nach Konflikten. Mit allen diesen Missionen kann zur Bekämpfung des Terrorismus beigetragen werden, unter anderem auch durch die Unterstützung für Drittländer bei der Bekämpfung des Terrorismus in ihrem Hoheitsgebiet.“

Dass nach Artikel 36 das Europäische Parlament zu Fragen der GASP „gehört werden soll und dass seine Auffassungen ... gebührend berücksichtig werden" sollen, es sogar Anfragen und Empfehlungen an den Rat richten darf, und zweimal jährlich eine Aussprache „über die Fortschritte bei der Durchführung der GASP“ führt, zeugt vom Demokratieverständnis des Lissabon-Vertrags und den sich darin bietenden Handlungsvollmachten der Regierungen.

Eine weitere Frage ist in diesem Zusammenhang von Bedeutung: Das Verhältnis der EU und ihrer GASP bzw. ESVP zur NATO. Diese ist ja nun spätestens seit dem am 24. April 1999 beschlossenen strategischen Konzept weltweit zuständig (NATO 1999). In Ziffer 19 des neuen Konzepts erfand sie die „NichtArtikel-Fünf-Krisenreaktions-Operationen“, die „gleiche politische und militärische Qualität" erfordern wie die klassischen Verteidigungsoperationen nach Artikel 5 des NATO-Vertrags. Vor allem: Die EU und ihre GASP wurden damals noch als zur NATO komplementäre Organisationen gesehen, wobei allerdings die NATO „das essentielle Forum für Konsultationen zwischen den Alliierten und das Forum für die Abstimmung ihrer Politiken“ bleiben soll (Ziffer 25). Dieser Konsens spiegelte noch die Angst Washingtons, die EU könne sich zu einer Parallelstruktur, ja zu einem Rivalen der US-dominierten NATO entwickeln (Asmus et al. 2010). Ganz anders die in Lissabon am 19. November 2010 beschlossene neue Strategie:

„Eine aktive und effektive Europäische Union trägt zur Sicherheit des euro-atlantischen Raumes bei. Deshalb ist die EU ein einzigartiger und wesentlicher Partner für die NATO. ... NATO und EU können und sollen komplementäre und sich gegenseitig stärkende Rollen spielen im Erhalt des internationalen Frieden und der Sicherheit.“ (Ziffer 32). (NATO 2010: 9)

Die EU und ihre GASP werden also nicht mehr als Konkurrenz zur NATO betrachtet. Hier soll nicht darüber spekuliert werden, ob diese Aufwertung der EU auch Ausdruck einer US-Außenpolitik sein könnte, die ihre Grenzen als Hegemon in einer zunehmend multipolaren Welt sieht und die EU als wichtigen Partner gegen neue aufsteigende Mächte wie China begreift und sie deshalb an sich binden möchte. Kurzum: Die Rolle der EU als globaler Sicherheitsakteur ist aufgewertet.

Festzuhalten ist: Deutschland hat bisher immer im Bündnis gehandelt, sei dies die NATO oder zunehmend die EU, und es wird dies auch weiterhin tun. Die einschlägigen Regelungen des Lissabon-Vertrages liefern hierfür geradezu ideale 
Voraussetzungen: Als wichtigste europäische Macht hat Deutschland dort entscheidenden Einfluss auf die Erteilung und die Ausgestaltung der Außenpolitik und auf gegebenenfalls zu erteilende Mandate für militärische Interventionen. Und: Im Verhältnis zum bisherigen alleinigen Hegemon gewinnt die EU - und damit Deutschland - an Gewicht. Selbstbewusst publizierte das Institut für Sicherheitsstudien der EU pünktlich zum NATO-Gipfel in Lissabon drei Studien (EUISS 2010a, b, c), in denen als erste Priorität gemeinsame EUStrategien für Afrika gefordert wurden. ${ }^{4}$

Für den außen- und sicherheitspolitischen Handlungsanspruch ergibt sich allerdings eine entscheidende Frage: In Deutschland gilt nach wie vor der Parlamentsvorbehalt, in der EU gilt dieser nicht. Ein diesbezügliches Urteil des Bundesverfassungsgerichts unterstreicht, dass nationales Recht weiter gilt, EU-Beschlüsse also die Mandatserteilung durch den Deutschen Bundestag nicht ersetzen können (BPB 2009). Dies könnte ein Hemmschuh für Deutschlands Bestreben sein, im Rahmen der EU wieder eine Weltgeltung zu erlangen, die ihm bisher versagt blieb. Dennoch dürften die jeweiligen zu erwartenden Entscheidungen des Deutschen Bundestages Beteiligungen an Militäreinsätzen kaum verhindern. Die Rückkehr zum Recht des Stärkeren unter Missachtung des Völkerrechts steht wieder auf der Tagesordnung auch für Deutschland.

\section{Deutschland und die Vereinten Nationen}

Inzwischen ist die Bundesrepublik zum vierten Mal für zwei Jahre nichtständiges Mitglied des Sicherheitsrates der Vereinten Nationen. Seit Mitte der 90er Jahre strebt Deutschland in den VN offen einen Ständigen Sitz mit der Begründung an, es wolle und müsse aufgrund seiner wirtschaftlichen und politischen Rolle in der Welt „mehr Verantwortung“ tragen. Diese Anläufe wurden jedoch bisher immer wieder erfolgreich von den fünf Ständigen Mitgliedern abgewehrt. Besonders absurd ist bei diesen Versuchen, dass, gelänge das deutsche Vorhaben, dann drei Mitgliedstaaten der EU Ständige Sitze im Sicherheitsrat innehaben würden. Doch das deutsche Streben macht Fortschritte. So ist die Bundesrepublik mittlerweile ihrem Ziel einen Schritt näher gekommen: Die Verhandlungen mit dem Iran über dessen Atomprogramm werden inzwischen offiziell geführt von „den fünf Ständigen Mitgliedern und Deutschland“.

Die aktive Rolle Deutschlands in den VN verfolgt aber noch ein zweites Ziel: Deutschland beharrt darauf, dass Militärinterventionen ausschließlich auf der Basis eines UN-Mandats durchgeführt werden dürfen. Das Fehlen eines solchen Mandats war zwar kein Grund, eine Beteiligung am NATO-Krieg gegen

4 Die EUISS-Studie „A Strategy for EU foreign policy“ fordert mit klaren Worten, dass die im Rahmen der GASP durchgeführten Missionen gemeinsame zivile und militärische Aktionen der EU in Afrika durchführen sollen. Die NATO wird hierbei nicht einmal erwähnt (EUISS 2010a, 59). 
Jugoslawien abzulehnen, es wurde jedoch benutzt, um die offizielle deutsche Beteiligung am Krieg gegen Irak zu verweigern.

Diese Doppelstrategie verfolgt ein wichtiges Ziel: Die Betonung der Rolle der VN - insbesondere ihres Sicherheitsrats - macht Deutschland zu einem wichtigen Akteur in der Weltorganisation, ganz gleich, ob es schließlich einen Ständigen Sitz erlangen kann oder nicht: Das Beharren auf der UNMandatierung von Militäreinsätzen stärkt vordergründig den Sicherheitsrat, indirekt aber auch Deutschland und sein Streben nach einem Ständigen Sitz in diesem Gremium. Andererseits hat sich der Sicherheitsrat inzwischen zu einer Art Selbstbedienungs-Institution für die Mandatsvergabe entwickelt: Er beschließt nicht nur Mandate, sondern Staatengruppen und Einzelstaaten treten an ihn heran, um ein Mandat zu erhalten und handeln dieses mit dem Sicherheitsrat aus. Hier nur zwei eklatante Beispiele: Die Beteiligung der deutschen Marine am UNIFIL-Mandat vom September 2006 zwecks Kontrolle der libanesischen Küste wurde in tagelangen zähen Verhandlungen zwischen der BRD und dem Sicherheitsrat erarbeitet (vgl. BAF 2006). Das zweite Beispiel ist der UN-mandatierte Militäreinsatz der EU im Tschad (EUFOR Tschad/RCA), den der Französische Außenminister Kouchner treffend so charakterisierte: „Diese französische Mission wird eine europäische sein“ - mit UN-Mandat, versteht sich.

Es ist daher absolut folgerichtig, Militäreinsatze nach Kap. VII der UN-Charta grundsätzlich abzulehnen, wie Jan van Aken dies in seinem Beitrag zur Programm-Debatte der LINKEN fordert (van Aken 2010). In der Tat: Einsätze nach Kap. VI der Charta, also im Rahmen der friedlichen Beilegung von Streit, sind zu befürworten, sind sie doch grundsätzlich nicht-militärisch, allenfalls das klassische Blauhelmkonzept ${ }^{5}$ wäre hier möglich. Anders verhält es sich mit Kampfeinsätzen nach Kap. VII, wo es um „Maßnahmen bei Bedrohung oder Bruch des Friedens und bei Angriffshandlungen“ geht. Statt ihre Blockadehaltung bzgl. des Art. 47 der Charta aufzugeben und dem Sicherheitsrat Truppen zur Verfügung zu stellen, lassen sich die Mitgliedsstaaten die Mandate für ihre eigenen Interventionen erteilen.

Damit versuchen die großen Staaten, ihre eigenen Interessen durchzusetzen, denn Militär ist Instrument der Politik und daher niemals neutral. Dies illustrieren nicht nur die beiden erwähnten Beispiele, sondern auch Afghanistan ebenso wie der Versuch der USA, ein UN-Mandat für den Angriff auf den Irak zu erhalten. Letztlich beschädigen diese Mandate, die sich die mächtigen Staa-

5 Da der Art. 47 der UN-Charta (Bereitstellung von Truppen der Mitgliedstaaten für den Sicherheitsrat) nie realisiert wurde, entwickelten die VN die sog. Blauhelme: Sie wurden nur im Einverständnis mit den Konfliktparteien stationiert; sie waren allenfalls für die Selbstverteidigung bewaffnet; vor allem: nur kleine und neutrale Staaten waren Truppensteller. Damit unterscheidet sich das alte, völkergewohnheitsrechtlich entwickelte Instrument der „Blauhelme“ radikal von der seit Ende der Bipolarität üblich gewordenen Praxis. 
ten zur Durchsetzung ihrer Interessen besorgen, das in der Charta angelegte supra-staatliche Gewaltmonopol. Dieses wird ausgehöhlt, und damit werden die Art. 2.4 (Gewaltverbot) und 2.7 (Nichteinmischungsgebot) der Charta völkergewohnheitsrechtlich außer Kraft gesetzt. Nicht um der Stärkung der VN willen, wie behauptet wird, sondern um hier gleichziehen zu können, strebt Deutschland nach einem Ständigen Sitz im Sicherheitsrat.

\section{Deutsche strategische Konzepte}

Während in der Opposition noch über die humanitäre Rechtfertigung von Militäreinsätzen gestritten wird, sind Regierung und Bundeswehr diesen Debatten längst voraus. Auslandseinsätze sollen - wie ja in der ESS, im LissabonVertrag und im strategischen Konzept der NATO gefordert - zur Normalität werden: Die Sprachregelung heißt jetzt „Armee im Einsatz“. Hierzu liegen zwei Grundsatzpapiere vor: Der im Oktober 2010 erschienene Bericht der sog. Weise-Kommission (BW 2010b) und der Bericht des Generalinspekteurs für die Bundesregierung (BW 2010a).

Die Weise-Kommission benennt im Titel der Studie klar die neue Denkrichtung des Bundesministeriums für Verteidigung: „Vom Einsatz her denken“. Die Umsetzung dieses Ziels soll durch „mehr Flexibilität und höhere Effizienz in der Bundeswehr erreicht werden (BW 2010b: 3). Es geht um eine „neue Bundeswehr“, die ganz offensichtlich mit den im Grundgesetz formulierten Aufgaben bricht und NATO und EU auf gleiche Ebene stellt. Nunmehr geht es um die „Erfüllung der sicherheitspolitischen und militärischen Zielvorgaben der NATO und der Europäischen Union“, wie sie in deren Sicherheitsstrategien festgeschrieben sind. Unmissverständlich wird - explizit darauf hingewiesen, dass die humanitären Einsätze der Vergangenheit angehören, geht es doch ab jetzt um „aktive Militäreinsätze ... von Somalia über das ehemalige Jugoslawien bis $\mathrm{zu}$ Afghanistan und Marineeinsätzen am Horn von Afrika.“

Ergänzt werden die Vorschläge der Weise-Kommission durch den fast zeitgleich vorgelegten Bericht des Generalinspekteurs der Bundeswehr. Dieser benennt im Detail die Reformvorstellungen. Daraus seien hier einige Kernaussagen zitiert, deren Sprache geradezu verblüffende Parallelen zur ESS aufweist. Der Umbau der Bundeswehr weg von der Berufsarmee ist unverzichtbar, denn „eine Schwerpunktverlagerung zu professionelleren Streitkräfte [ist] sicherheitspolitisch unabdingbar." Die Bundeswehr soll globaler Akteur werden und „auch auf das Unvorhergesehene reagieren können.“ (BW 2010a: 13)

„Die Einsatzrealität der vergangenen zwanzig Jahre und Zukunftsanalysen zeigen, dass Risiken und Bedrohungen von Staaten und Regionen ausgehen können, die sich der Kontrolle durch ein funktionierendes Staatswesen entziehen. Dazu gehören die Gebiete scheiternder und gescheiterter Staaten, die Hohe See, der Luftraum über diesen Regionen, der Weltraum und große Teile des Informationsraumes." (BW 2010a: 12) 
Die Bundeswehr schrumpft von derzeit noch 252.000 Soldatinnen und Soldaten im Jahre 2005 auf 166.000, 96\% davon sollen Berufssoldaten sein.

So soll die Armee „effizienter, schlagkräftiger, moderner“ werden, um mit ihren Fähigkeiten zur Spitze der Bündnisse zu gehören. Diesem Ziel folgt der aufschlussreiche Satz „Hier setzen jedoch nationale Souveränitätserwägungen durchaus Grenzen." (BW 2010a: 22) Damit wird eine Art nationaler Vorbehalt formuliert, der den Verpflichtungen in den Bündnissen dann und dort Grenzen setzt, wo das nationale Interesse der Bundesrepublik berührt werden könnte.

Die Zielvorgaben für die Streitkräfte der Zukunft sind:

- „Dauereinsätze mit mindestens 10.000 (statt bislang rund 7.000) Soldatinnen und Soldaten in mehreren Einsatzgebieten, dabei

- Vollunterstützung der Anteile Land- und Luftstreitkräfte in zwei Einsatzgebieten, mit ggf. zusätzlicher kommerzieller Unterstützung oder Host Nation Support,

- davon unabhängig organische und kommerzielle Unterstützung der Seestreitkräfte." (BW 2010a: 24)

Hier werden die Vorgaben der ESS ins Operative übersetzt. Auch geht es nicht mehr um „Streitkräfte zur Verteidigung“, sondern um Kampfeinsätze, und dies weltweit. Folgerichtig findet die Notwendigkeit einer Mandatierung durch die VN keine Erwähnung - auch wenn davon auszugehen ist, dass diese für die unmittelbare und mittelfristige Zukunft eingeholt werden dürften. Die „humanitäre Begründung“ ist obsolet geworden und findet auch keine Erwähnung als Begründung für Einsätze.

Ganz im Zuge neoliberaler Verschlankung soll in Zukunft auch auf private militärische Dienstleister zurückgegriffen werden. Schließlich wird dem nationalen Alleingang ein Türchen geöffnet, setzen doch „nationale Souveränitätserwägungen“ dem Handeln in den Bündnissen „durchaus Grenzen“. Damit ist die Bundeswehr aufgestellt für Militäreinsätze jeder Art, bündnispolitische oder geografische Beschränkungen gibt es nicht mehr.

\section{Afrika - ein Exerzierplatz?}

Mit der Ausrufung des ,global war on terrorism“ am 20. September 2001 hatten die USA nach den Anschlägen des 11. September die „Operation Enduring Freedom" (OEF) ins Leben gerufen (Dep. of State 2001). Das Operationsgebiet von $\mathrm{OEF}$ erstreckte sich von Afghanistan über das Horn von Afrika und - unter Verweis auf die Anschläge in Kenia - bis Tansania und in den Sahel. Der Terrorismus in diesem Raum wurde auch beschworen im Zusammenhang mit der Entführung von 32 Europäern in der algerischen Sahara im Jahre 2003. Unter implizitem Verweis auf diesen Akt gründeten die USA im Februar 2007 ein eigenes Kommando für Afrika, African Command, kurz: Africom (Ruf 2008). Es schließt den Ring der den Globus umspannenden amerikanischen Kommandos, die parallel zur und unabhängig von der NATO existie- 
ren. Der afrikanische Kontinent ist seit jüngstem neben den asiatischen Ölund Gasfeldern ins Fadenkreuz der großen Mächte geraten, da dort Lagerstätten des Schwarzen Goldes noch nicht voll erschlossen sind und weitere Reserven vor allem im Sahel-Raum und im Golf von Guinea vermutet werden. Afrika birgt weitere energetische Ressourcen wie Uran, das seit Peak Oil als Energiequelle immer wichtiger wird, aber auch Gold, Diamanten, Kupfer und das für Informationstechnologien unerlässliche Coltan. Um ihre Energieabhängigkeit von den asiatischen Staaten zu reduzieren, erklärten die USA im Jahre 2007 (FAZ, 24.4.2007), dass sie ihre Importe aus Afrika von damals 13\% auf 25\% im Jahre 2013 steigern wollten und dass die Carter-Doktrin ${ }^{6}$ hinfort auch für Afrika gelte (Volman 2006).

Neben den alten Kolonialmächten und den USA hat sich in Afrika China ${ }^{7}$ als neuer Akteur etabliert. Dies gilt in herausragender Weise für Sudan, dessen Öl zu 63\% nach China verschifft wird, aber auch für die ölreichen Länder Nigeria und Angola (Kappel/Schneidenbach 2006). Derzeit ist China dabei, sich in Niger zu engagieren, das über die drittgrößten Uran-Reserven der Welt verfügt. Dieses bitterarme Land, ehemalige französische Kolonie, ist fest in der Hand von Areva, der wohl weltgrößten Firma im Bereich von Uran-Abbau, Atomanlagenbau und Wiederaufbereitungsanlagen (Ruf 2009). Die Interessen der EU wie der USA liegen also zum Einen in der Ausbeutung der gigantischen Rohstoffreserven des Kontinents, wobei durchaus Rivalitäten zwischen beiden bestehen, zum Andern aber ist es in beider Interesse, die politische Kontrolle über die afrikanischen Staaten so zu gestalten, dass der Einfluss Chinas auf dem Kontinent eingedämmt werden kann.

Angesichts dieser geopolitischen Lage ist es kein Zufall, dass auch Deutschland sich zunehmend in Afrika engagiert. Zwar entfällt die höchste Zahl der derzeit eingesetzten deutschen Soldatinnen und Soldaten auf Afghanistan, und von der Zahl der Einsätze her liegt der Schwerpunkt bisher auf dem Balkan. Aber: In Afrika beteiligt(e) sich Deutschland seit 1993 an zwölf Einsätzen. Die höchsten Truppenstärken erreichte der UN-mandatierte Einsatz der UNOSOM in Somalia, wo von 1993 bis 19941.700 Heeressoldaten und 600 Marinesoldaten eingesetzt waren, hinzu kamen 120 Soldaten der Luftwaffe in Kenia. Der unter quantitativen Gesichtspunkten zweitwichtigste Einsatz ist die Operation ATALANTA/EU NAVFOR im Golf von Aden (seit 2008) zum

6 In Reaktion auf die iranische Revolution hatte der damalige US-Präsident Jimmy Carter am 23. Januar 1980 vor dem Kongress erklärt: "Jeder Versuch einer fremden Macht, die Kontrolle über die Region am Persischen Golf zu erlangen, wird als Angriff auf die lebenswichtigen Interessen der Vereinigten Staaten angesehen. Jeglicher Angriff dieser Art wird mit allen Mitteln zurückgeschlagen werden, auch mit militärischen."

7 Aufschlussreich sind auch die Berichte der US-Botschaften in Afrika zur Rolle Chinas auf dem Kontinent, vgl. „Pas une menace... mais à surveiller de très près“, in: El Watan (Algier), 14.12.2010. 
Schutz vor somalischen Piraten, die von der EU mandatiert ist. Auf deutschen Kriegsschiffen sind dort bis zu 1.400 Soldaten im Einsatz. Ein weiterer personalintensiver Einsatz der Bundeswehr war die Mission EUFOR in der Demokratischen Republik Kongo, die vom 3. Juli bis 30. November 2006 die Präsidentschaftswahlen in diesem Land sichern sollte. Das Oberkommando dieser Operation war in Potsdam. Der weitaus überwiegende Teil der deutschen Beteiligungen ist wenig personalintensiv und beschränkt sich meist auf Unterstützung, Transport, Logistik und bisweilen Ausbildung oder Beobachtung von Waffenstillständen (EU 2007). Präsenz zeigen, beteiligt sein ist wichtig, will man an Entscheidungen teilhaben.

Auch die Mandatierung lässt Schlussfolgerungen zu: Von den erwähnten zwölf Einsätzen basieren sechs auf einem Mandat des UN-Sicherheitsrats. Drei sind von der EU mandatiert. Jedoch: Zwei weitere Missionen (die Unterstützung der MONUC im Kongo und die EUTM in Somalia/Uganda) sind gleichfalls von der EU mandatiert. Für die Erstellung des Mandats stützte sich die EU auf Resolutionen des Sicherheitsrats, die sie so interpretierte, dass die Mandatserteilung möglich wurde. Damit wird die oben aufgestellte These belegt, dass die EU sich zunehmend selbständig Mandate erteilt, indem sie auf Sicherheitsratsresolutionen verweist. Da es sich bei MONUC und EUTM um Mandate handelt, die der Sicherheitsrat für von der Afrikanischen Union (AU) geführte Operationen wie AMISOM oder MONUC erteilte, lässt sich schließen, dass die EU sich in diese Missionen einschaltet, um dabei eigene Interessen zu verfolgen. Offizielle Begründung ist, die EU wolle den meist ungenügend ausgerüsteten Kräften der AU logistische Hilfe leisten. Dieses Argument ist sicherlich zutreffend, jedoch: Die EU könnte der AU auch Material und Logistik unter ausschließlichem Kommando der AU zur Verfügung stellen - ohne Beteiligung an einem bestehenden Mandat oder Einrichtung eines eigenen Mandats.

Nicht erwähnt ist in der vorstehenden Aufzählung der Mandate die Mission EUFOR Tschad/RCA, die aufgrund eines UN-Mandats nominell von der EU, in Wirklichkeit von Frankreich geführt wurde und an der die Bundeswehr nicht beteiligt war, dafür aber 18 andere europäische Staaten, darunter Österreich mit rund 200 Soldaten. Diese Mission (März 2008 bis März 2009) war politisch hoch umstritten. ${ }^{8}$ Ebenfalls nicht erwähnt ist die die deutsche Beteiligung an der Operation Enduring Freedom (OEF), da sie von keiner Resolution des Sicherheitsrats gedeckt war. Dennoch waren an ihr laut Mandat des Deutschen Bundestages bis zu 3.900 Soldaten beteiligt (Bundestag Drucksache 14/7296, 7.11.2001). Die Grundlage hierfür war das Kanzler-Wort von der „uneingeschränkten Solidarität“ nach den Anschlägen von 9/11. Die Zahl der

8 Die ehemalige Kolonialmacht des Tschad (und der Zentralafrikanischen Republik), Frankreich, war bereits mit einer eigenen Truppe von insgesamt 1.100 Soldaten im Tschad präsent und unterstützte im dortigen Bürgerkrieg massiv den Präsidenten Déby (Tull 2008; Ehrhart 2008). 
im Rahmen von OEF bereit gestellten deutschen Truppen wurde zunehmend abgesenkt, die Beteiligung im Juni 2010 beendet. (BT 2010)

Afrika ist für die Bundeswehr also nicht Exerzier- oder Truppenübungsplatz im klassischen Sinne, sondern ein wichtiges politisches und geostrategisches Feld. Die Bedeutung Afrikas liegt in seinem Rohstoffreichtum, Dabei ist die Sicherung der Transportwege mindestens ebenso wichtig wie die der Lagerstätten. Sie erfolgt vorzugsweise durch die Stützung „freundlicher“ Regime. Dies illustriert die Politik Frankreichs und der EU in der weit überwiegenden Zahl der ehemaligen französischen, aber auch belgischen oder britischen Kolonien. (Ruf 2010a, b) Die geostrategischen Zusammenhänge werden deutlich, wenn man weiß, dass das Öl des Tschad mittels einer Pipeline durch die gleichfalls ehemalige französische Kolonie Kamerun zum Golf von Guinea transportiert wird, oder dass im Jahr 2010 mit dem Bau einer Gas-Pipeline begonnen wurde, die über $4.000 \mathrm{~km}$ von Nigeria an die algerische Mittelmeerküste geführt wird. Dass der Sahel-Raum und die Sahara zentrales Aktionsgebiet von Africom aber auch der EU sind (siehe EUFOR Tschad/RCA), ist daher nicht verwunderlich.

Afrika verspricht, in den nächsten Jahren zu einem, ja möglicherweise zu dem Hauptschauplatz von Militäreinsätzen zu werden. Nicht nur schreitet die (zivile, vor allem entwicklungspolitische!) chinesische Durchdringung des Kontinents rapide voran, die EU (vor allem Frankreich) verteidigt dort „angestammte" Interessen und bestimmt weitgehend die inneren Machtstrukturen der Länder. Mit US-Africom sind nun auch die USA militärisch präsent. Dies heißt nicht, dass etwaige Rivalitäten in militärische Auseinandersetzungen umschlagen müssen. Aber die innenpolitische Situation in den ohnehin durch prekäre Staatlichkeit gekennzeichneten Ländern könnte in den Sog dieser Rivalitäten geraten. Die Unterstützung herrschender Regierungen gegen Rebellen (oder umgekehrt) ist Teil dieser Kämpfe um Einflussnahme der großen Akteure. Schon jetzt tragen sie zu wachsender Instabilität bürgerkriegsähnlichen Auseinandersetzungen bei (Tschad, Elfenbeinküste, Sudan, Niger, Kongo, Mauretanien ...).

Dies alles geschieht auf einem Kontinent, dessen Länder gewaltige Ressourcen besitzen, zugleich aber zu den ärmsten der Welt zählen, auf einem Kontinent, auf dem die Folgen des Klimawandels zu weiterer Versteppung und Ausdehnung der Wüsten, verbunden mit der Zerstörung noch vorhandener Reste von Subsistenzproduktion führen. Die jüngste Ausbreitung von land grabbing, (Bohne 2010; Avril 2010) der Aneignung großer agrarischer Flächen durch Staaten (darunter China) und Konzerne bringt neue und extrem brutale Formen der ursprünglichen Akkumulation und weitere Migrationsströme hervor. Die in der ESS benannten Ursachen von Konflikten, Armut, Hunger und Elend werden rasant ansteigen.

Vor dem Hintergrund wachsender Konflikthaftigkeit, die durch die Interessen der großen Mächte noch geschürt wird, dürfte der geschundene Kontinent in 
noch höherem Maße als schon bisher zum Schauplatz militärischer Interventionen werden - sei es mit oder ohne Mandat der UN. Das alles spricht dafür, dass die EU - unabhängig vom Sicherheitsrat - ihrerseits Interventionen durchführen wird, so wie der „Antiterrorkampf“ der USA unabhängig von Mandaten der UN weitergehen wird. All dies geschieht zu einem Zeitpunkt, in dem die NATO und ihre Führungsmacht, die USA, in ihrem Lissabonner Strategiekonzept vom November 2010 erstmals die EU als „einzigartigen und wesentlichen" Partner anerkannt haben (Ziffer 32).

Hier schließt sich der Kreis: Die imperialen Ambitionen der EU unterscheiden sich nicht mehr von denen der USA. Inwieweit ihr langer - und erfolgreicher Kampf um (gleichberechtigte) Anerkennung durch die USA auch Ausdruck des imperialen Niedergangs der USA sein könnte, muss dahingestellt bleiben. Festzuhalten bleibt: Der Kompetenzzuwachs der EU bedeutet zugleich die Stärkung Deutschlands innerhalb der EU. So ist die schwächste und ärmste, aber extrem rohstoffreiche Weltregion der ideale Ort für die Verwirklichung alter und neuer Großmachtziele. Für Deutschland resultiert eine zunehmende aktive Beteiligung an EU-Militäreinsätzen sowohl aus seinen wirtschaftlichen Interessen wie aus der Logik seiner militärpolitischen Emanzipation. Der Umbau der Bundeswehr zur „Armee im Einsatz“ ist Beleg für die Aktualität dieses Politikziels. Dass die Alliierten den martialischen Tönen aus Berlin applaudieren, belegt zweifelsfrei: Wir sind wieder wer - endlich auch militärisch.

\section{Literatur}

Asmus, Ronald; Czmur, Stefan; Donnelly, Chris; Ronis, Aivis; Valasek, Tomas; Wittmann, Klaus (2010): NATO, new allies and reassurance, Center for European Reform - policy brief, http://www.cer.org.uk/pdf/pb_nato_12may10.pdf (08.12. 2010).

Avril, Hilaire (2010): Africa: Land Grabs Continue as Elites Resist Regulation, http://ipsnews.net/newsTVE.asp?idnews=51018 (18.04.10).

BAF - Bundesausschusses Friedensratschlag (2006): "Die Politik dankt ab und die Marine läuft aus" Sechs Gründe der Friedensbewegung gegen den Bundeswehreinsatz im Libanon, Kassel, http://www.ag-friedensforschung.de/regionen/Libanon/baf-presse2.html (28.1.2011).

Bohne, Andreas (2010): „Land Grabbing“ in Afrika, http://entwickler-netz.de/blog/2010/01/11/ $\%$ E2\%80\%9Eland-grabbing\%E2\%80\%9C-in-afrika/ (13.12.2010).

BPB - Bundeszentrale für politische Bildung (2009): Urteil des Bundesverfassungsgerichts zum Vertrag von Lissabon, http://www.bpb.de/themen/NIPB3X,0,0,Urteil_des_Bundesverfassungs gerichts_zum_Vertrag_von_Lissabon.html (8.12.2010).

BW - Generalinspekteur der Bundeswehr (2010a): Bericht zum Prüfauftrag aus der Kabinettsklausur vom 7. Juni 2010, in der Endfassung vom 31. August 2010, http://www.bmvg.de/fileserving/Portailes/C1256EF40036B05B/W288WCHU749INFODE/Bericht $\% 20 \mathrm{des} \% 20$ GenInsp $\%$ 20\%20Endfassung\%20\%20310810.pdf (10.12.2010).

BW - Strukturkommission (2010b): Bericht „Vom Einsatz her denken“ (Oktober 2010), http://www.bmvg.de/fileserving/PortalFiles/C1256EF40036B05B/W28AL8JU967INFODE/Ber icht $\% 20 \mathrm{der} \% 20$ Strukturkommission $\% 20 \mathrm{der} \% 20$ Bundeswehr.pdf (10.12.2010).

BT - Bundestag (2010): OEF/OAE (Horn von Afrika/Mittelmeer), http://www.bundestag.de/ bundestag/ausschuesse17/a12/auslandseinsaetze/auslandseinsaetze/oef.html (12.12.2010). 
Department of State (2001): Bush Announces Start of a "War on Terror". President Addresses Joint Session of Congress Sept. 20, http://www.globalsecurity.org/military/library/news/ 2001/09/mil-010920-usia01.htm (10.12.2010).

Ehrhart, Hans-Georg (2008): Die Operation EUFOR Tschad/ZAR: Werte, Ziele, Interessen, Probleme, in: Gießmann, Hans J.; Neuneck, Götz (Hg.): Streitkräfte zähmen, Sicherheit schaffen, Frieden gewinnen. Festschrift für Reinhard Mutz, Baden-Baden, 159-170.

EU (2003): Ein sicheres Europa in einer besseren Welt. Europäische Sicherheitsstrategie, Brüssel, http://www.consilium.europa.eu/uedocs/cmsUpload/031208ESSIIDE.pdf (28.1.2011).

- (2008): Konsolidierte Fassungen des Vertrags über die Europäische Union und des Vertrags über die Arbeitsweise der Europäischen Union, Brüssel, http://www.consilium.europa.eu/ uedocs/cmsUpload/st06655-re01.de08.pdf (28.1.2011).

EU - European Council (2007): EUFOR RD Congo. Factsheets, http://www.consilium. europa.eu/showPage.aspx?id=1095\&lang=en (08-12-10)

EUISS - European Union Institute for Security Studies (2010a): A Strategy for EU foreign policy, Paris http://www.iss.europa.eu/uploads/media/A_strategy_for_EU_foreign_policy.pdf (28.1.2011).

- (2010b): Quelle Défense Européenne en 2020?, Paris.

- (2010c): What do Europeans want from NATO, Paris.

Kappel, Robert; Schneidenbach, Tina (2006): China in Afrika, GIGA-Focus Nr. 12/2006. http://www.giga-hamburg.de/content/publikationen/pdf/gf_global_0612.pdf (12.12.2010).

Kutscha, Martin (2010): Verfassungs-Streich; in: Forum Wissenschaft Nr. 3/2010, 4-7.

Nato (1999): The Alliance's Strategic Concept, http://www.nato.int/cps/en/natolive/ official_texts_27433.htm (8.12.2010).

- (2010): Strategic Concept For the Defence and Security of The Members of the North Atlantic Treaty Organisation. Active Engagement, Modern Defence, Lisbon, http://www.nato.int/lisbon2010/strategic-concept-2010-eng.pdf (8.12.2010).

Ruf, Werner (2008): Geopolitik und Ressourcen: Der Griff der USA nach Afrika, in: ÖSFK/Roithner, Thomas (Hg.): Von kalten Energiestrategien zu heißen Rohstoffkriegen? Münster, 160-173.

Ruf, Werner (2009): Ein noch nicht mediatisierter Konflikt: Niger, in: Ruf et al.: Militärinterventionen: Verheerend und völkerrechtswidrig, Berlin, 191-203.

- (2010a): Gelockerte Fesseln, in: Junge Welt, 24. August.

- (2010b): Plünderökonomie; in: Junge Welt, 25. August.

Schmähling, Elmar (1990): Der unmögliche Krieg. Sicherheit und Verteidigung vor der Jahrtausendwende, Düsseldorf 1990.

Tull, Denis (2008): Tschad-Krise und die Operation EUFOR Tschad/ZAR, SWP-Aktuell, 15. Februar, http://www.swp-berlin.org/produkte/swp_aktuell_detail.php?id=8771 (10.12.10).

van Aken, Jan (2010): Über die friedliche Beilegung von Streitigkeiten, in: Neues Deutschland, 1. November 2010.

Volman, Daniel (2006): The Scramble for African Oil, in: New African, Juli 2006, 20.

Ziegler, Jean (2005): Das Imperium der Schande, München. 